14.03

\title{
О возможном влиянии факторов урбанизации на распространенность сахарного диабета первого типа на территории РФ
}

\author{
(ㄱ Л.А. Сопрун, ${ }^{1}$ С.Г. Ястребов ${ }^{2}$ \\ ${ }^{1}$ Санкт-Петербургский государственный университет, медицинский фракультет, \\ 199106 Санкт-Петербург, Россия \\ ${ }^{2}$ Физико-технический институт им. А.Ф. Иофрфе РАН, \\ 194021 Санкт-Петербург, Россия \\ e-mail: lidas7@yandex.ru, yastrebov@mail.ioffe.ru
}

Поступило в Редакцию 22 января 2020 г.

В окончательной редакции 20 февраля 2020 г.

Принято к публикации 2 апреля 2020 г.

\begin{abstract}
С помощью метода выделения главных компонент, связанных с коэффициентами корреляции матрицы Пирсона, выявлена возможная связь между распространенностью сахарного диабета первого типа и различными факторами урбанизации на территории Российской Федерации. Создана гипотетическая физическая модель воздействия факторов урбанизации на заболеваемость. Решается задача выявления скрытых причин заболеваемости сахарным диабетом первого типа в различных регионах Российской Федерации и вклад факторов урбанизации в заболеваемость. Для анализа использованы разнообразные статистические данные по таким факторам урбанизации, как автомобильно-дорожный комплекс, выбросы в поверхностные сточные воды и в атмосферный воздух.
\end{abstract}

Ключевые слова: сахарный диабет первого типа, метод выделения парных главных компонент, ультрафиолетовое излучение, урбанизация.

DOI: $10.21883 /$ JTF.2020.09.49684.28-20

\section{Введение}

Как известно, урбанизация для человечества является как благом, так и разрушителем. Для того чтобы окончательно определиться в этом вопросе или начать новую полемику, необходимо разобраться с основными факторами, способствующими распространению и развитию различной патологии на территории РФ. Нами была взята за основу заболеваемость сахарным диабетом первого типа (DM1), как яркий пример аутоиммунной патологии, которая, в свою очередь, согласно теории калейдоскопа аутоиммунитета, имеет корреляционную взаимосвязь с окружающей средой. Напомним, что DM1, широко известный, как диабет, представляет собой группу метаболических нарушений, проявляющихся из-за недостаточности инсулина и характеризующихся высоким уровнем сахара в крови в течение длительного периода времени. Причины DM1 неизвестны, хотя было выявлено несколько факторов риска. Риск развития DM1 увеличивается при определенных вариантах генов HLA-DQA1, HLA-DQB1 и HLA-DRB1. Эти гены предоставляют инструкции для создания белков, которые играют важную роль в иммунной системе. На экспрессию этих генов может оказывать влияние дефицит витамина D. Действительно, в последнее время в литературе дефициту витамина D уделяется значительное внимание как к триггеру проявления этого заболевания. Отметим также, что длины волн УФ излучения Солнца, попа- дающие в спектральный диапазон от 290 до $300 \mathrm{~nm}$, являются наиболее эффективными для выработки витамина D3 [1-3].

В настоящей работе с использованием численных методов, изложенных в работе [2], выполнен анализ целого ряда статистических данных, представленных в [4], для различных субъектов Российской Федерации.

\section{1. Материалы и методы исследования}

Интересно выполнить анализ заболеваемости DM1 по набору доступных данных, в который входят источники антропогенной пыли, связанной с автомобильнодорожным комплексом (число автобусов общего пользования, плотность дорог, имеющих твердое покрытие, которое, в свою очередь, и является главным источником твердых пылевых частиц различного размера), а также с выбросами в атмосферный воздух и сбросами сточных вод в поверхностные водоисточники.

Исследование охватывало всю территорию РФ Центральный, Северо-Западный, Южный, Северо-Кавказский, Приволжский, Уральский, Сибирский, Дальневосточный Федеральные округа - по 73 регионам, входившим в состав РФ с года начала исследования [4]. Для обозначения региона мы используем следующие сокращения: Белгородская область - BelO, Брянская область - BrjaO, Владимирская область - VladO, Boронежская область - VorO, Ивановская область - IvO, 
Калужская область - $\mathrm{KalO}$, Костромская область $\mathrm{KosO}$, Курская область - KurO, Липецкая область $\mathrm{LipO}$, Московская область - MosO, Орловская область - OrlO, Рязанская область - RjazO, Смоленская область - SmolO, Тамбовская область - TambO, Тверская область - TverO, Тульская область - TulO, Ярославская область - JarO, город Москва - Msk, Республика Карелия - Kar, Республика Коми - KomR, Архангельская область - ArO, Вологодская область VolgO, Калининградская область - KalO, Ленинградская область - LenO, Мурманская область - MurmO, Новгородская область - NoagO, Псковская область - $\mathrm{PsO}$, город Санкт-Петербург - SPb, Республика Адыгея RAdg, Республика Калмыкия - ZKlm, Краснодарский край - Kdk, Астраханская область - AsO, Волгоградская область - VO, Ростовская область - RosO, Республика Башкортостан - RBsh, Республика Марий Эл - RMJe, Республика Мордовия - RMor, Республика Татарстан - RTts, Удмуртская Республика - RU, Чувашская Республика - RChu, Пермский край Permk, Кировская область - KirO, Нижегородская область - NGO, Оренбургская область - Oro, Пензенская область - PenO, Самарская область - SamO, Саратовская область - SarO, Ульяновская область U1O, Курганская область - KurO, Свердловская область - SverO, Тюменская область - TјumO, Челябинская область - ChelO, Республика Алтай - Ral, Республика Бурятия - RBu, Республика Тыва - Rty, Республика Хакасия - RНа, Алтайский край - AlK, Забайкальский край $-\mathrm{ZbK}$, Красноярский край $\mathrm{KdK}$, Иркутская область - IrO, Кемеровская область $\mathrm{KemO}$, Новосибирская область - $\mathrm{NsO}$, Омская область - OmO, Томская область - ТomO, Республика Саха (Якутия) - RSJa, Камчатская край - KamK, Приморский край - PrimK, Хабаровский край - HabK, Амурская область - AmO, Магаданская область $\mathrm{MagO}$, Сахалинская область - SahO, Еврейская автономная область - EvrAO, Чукотский автономный округ - ChAО. Для обозначения факторов урбанизации нами введены следующщие сокращения. Обозначения Сахарный диабет 1 типа (далее DM1), число автобусов общего пользования (далее $\mathrm{BS}$ ), дороги общего пользования, имеющее твердое покрытие (далее RD), выбросы сточных вод в поверхностные водоисточники (далее WW) и выбросы в атмосферный воздух от стационарных источников (далее АР) относятся соответственно к факторам заболеваемости DM1 (измеряется числом заболевших DM1 на 100000 человек), числу автобусов (на 100000 человек населения), протяженности (,плотности“) дорожного покрытия (измеряется в километрах дорог на 1000 квадратных километров территории) и выбросам в атмосферу (измеряются в тысяч тонн).

Предварительно данные для всех факторов урбанизации характеризовались простейшими статистическими методами, выполнялся тест на их соответствие нормальному закону распределения, строились гистограммы, представленные на рис. 1. Коэффициенты корреля-
Таблица 1. Описательная статистика

\begin{tabular}{l|c|c|c}
\hline Факторы & $\mathrm{N}$ & Значения & $\begin{array}{c}\text { Стандартные } \\
\text { отклонения }\end{array}$ \\
\hline $\mathrm{DM}$ & 73 & 15.553 & 9.842 \\
$\mathrm{BS}$ & 73 & 125.218 & 56.846 \\
$\mathrm{RD}$ & 73 & 197.617 & 178.567 \\
$\mathrm{WW}$ & 73 & 133.503 & 172.002 \\
$\mathrm{AP}$ & 73 & 201.491 & 154.092
\end{tabular}

ции $\left(r_{x y}\right)$ интерпретировались, исходя из абсолютных значений самого коэффициента корреляции Пирсона. Оценка статистической значимости коэффициента корреляции $r_{x y}$ осуществлялась при помощи t-критерия, рассчитываемого по следующей формуле:

$$
t_{r}=\frac{r_{x y} \sqrt{n-2}}{\sqrt{1-r_{x y}^{2}}} .
$$

Все полученные зависимости асимметричны, из чего следует отличие полученных гистограмм от нормального закона распределения. Данные описательной статистики приведены в табл. 1. Из таблицы видно, что среднеквадратичное отклонение для целого ряда факторов урбанизации, таких как $\mathrm{RD}, \mathrm{WW}, \mathrm{AP}$, представляют величины порядка средних значений.

Меньшее стандартное отклонение указывает на то, что больше данных сгруппировано относительно среднего значения. Большее, в свою очередь, указывает, что данные более распространены.

Затем методом Пирсона рассчитывались парные коэффициенты корреляции. Из табл. 1 видна положительная корреляция между всеми исследованными факторами урбанизации и DM1. Наибольшей является корреляция между числом автобусов и DM1.

Далее с помощью численных рецептов, приведенных в [5], проводится выделение главных компонент с использованием коэффициентов корреляционной матрицы, рассчитываются и приводятся кривые осыпи и рисунки счетов и нагрузок, выполненные в координатах выделенных главных компонент. Проводится анализ взаимного положения выделенных векторов друг относительно друга и относительно осей главных компонент. Сравнение позволяет сделать вывод о наличии скрытого фактора, влияющего на заболеваемость сахарным DM1, а именно недостатку инсоляции пациентов, приводящего к дефициту витамина D в ряде областей РФ. Известно, что в настоящее время дефицит витамина $\mathrm{D}$ рассматривается в качестве фактора риска, провоцирующего развитие этого опасного заболевания (см., например, [6]).

Можно предположить, что УФ излучение в этом спектральном диапазоне поглощается углеродной составляющей антропогенной пыли. Так, известно, что полициклические ароматические углеводороды являются компонентой такой пыли [7] и способны поглощать 

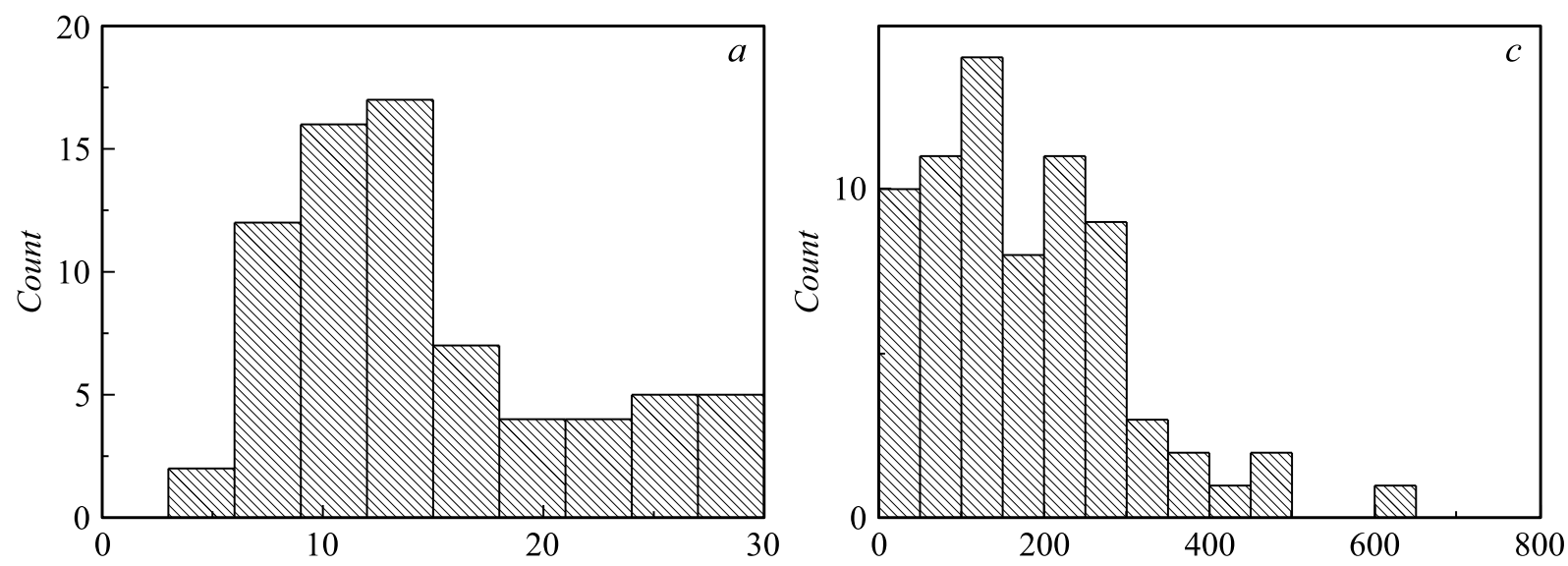

First type Diabetes mellitus, N/100000 inhabitants
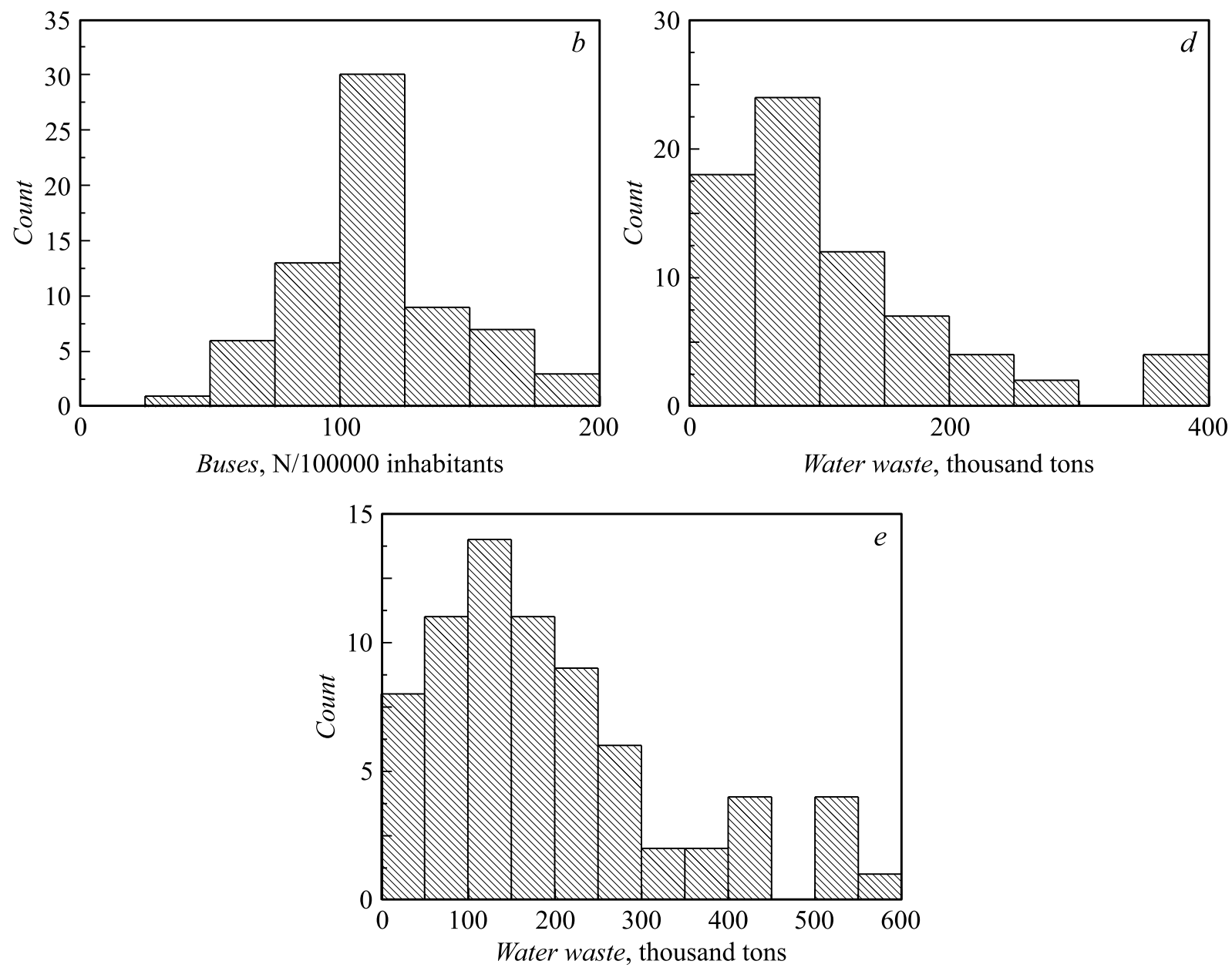

Рис. 1. $a$ - распределение заболеваемости DM1 в регионах России; $b-$ распределение относительного количества автобусов в регионах России; $c$ - распределение плотности дорог с твердым покрытием в регионах России; $d-$ распределение сброса сточных вод в регионах России; $e-$ распределение выбросов в атмосферу в регионах России.

УФ излучение актуального диапазона длин волн [8], т.е. наличие такой пыли в атмосфере способно поглощать УФ излучение Солнца и приводить к уменьшению инсоляции пациентов, что, в свою очередь, может способствовать D-витаминной недостаточности. Как следствие, возможно развитие DM1.

\section{2. Результаты}

Разные факторы урбанизации предварительно анализировались методами простой описательной статистики, данные которой приведены в табл. 1. Из таблицы видно, что стандартное отклонение превышает среднее 


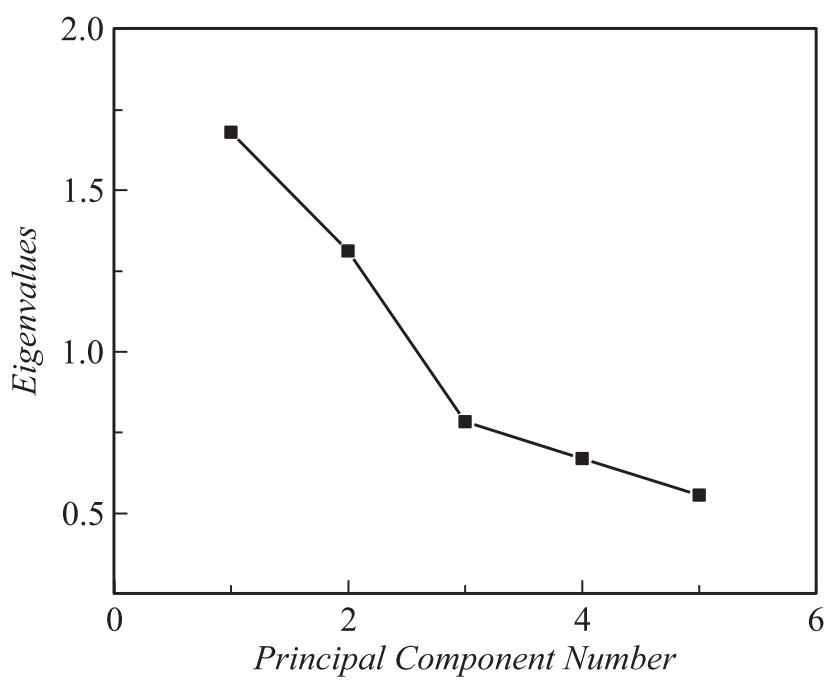

Рис. 2. Кривая осыпи.

Таблица 2. Корреляционная матрица Пирсона со значениям $r_{x y}$

\begin{tabular}{l|l|c|c|c|c}
\hline Характеристики & $\mathrm{DM}$ & $\mathrm{BS}$ & $\mathrm{RD}$ & $\mathrm{WW}$ & $\mathrm{AP}$ \\
\hline $\mathrm{DM}$ & 1 & 0.334 & 0.217 & 0.142 & 0.209 \\
$\mathrm{BS}$ & 0.334 & 1 & -0.023 & 0.071 & 0.317 \\
RD & 0.217 & -0.023 & 1 & 0.354 & -0.033 \\
WW & 0.142 & 0.071 & 0.354 & 1 & 0.054 \\
AP & 0.209 & 0.317 & -0.033 & 0.054 & 1
\end{tabular}

значение для сточных вод, что может быть связано с отличием распределения от нормального закона. Об отклонении от нормального закона также свидетельствует вид распределений (гистограмм), построенных для разных факторов и представленных на рис. 1.

Далее рассчитывались компоненты корреляционной матрицы Пирсона, которые представлены в табл. 2. Из таблицы следует, что DM1 положительно коррелирует со всеми исследованными факторами.

С целью дальнейшего анализа мы использовали метод выделения главных компонент, связанных с парными коэффициентами матрицы корреляций Пирсона. В табл. 3 приведены собственные значения матрицы Пирсона и соответствующий им процент частной доли и кумулятивного вклада в описание дисперсии.

Эти собственные числа в функции их порядкового номера приведены на рис. 2, этот рисунок называется кривой осыпи [5], а хорошим приближением в описании дисперсии считается удержание в последующем анализе тех собственных чисел матрицы Пирсона, которые лежат слева от точки излома (включая саму точку излома). Т.е. в рассматриваемом случае на полноту описания претендуют три собственных числа, которым соответствуют три главных компонента. Далее для интерпретации данных используется построение рисунков, называемых биплотами.
Таблица 3. Собственные значения матрицы Пирсона и соответствующий им процент частной доли и кумулятивного вклада в описание дисперсии

\begin{tabular}{c|c|c|c}
\hline № & Значения & Процент дисперсии, \% & Совокупность, \% \\
\hline 1 & 1.68111 & 33.62 & 33.62 \\
2 & 1.31269 & 26.25 & 59.88 \\
3 & 0.78262 & 15.65 & 75.53 \\
4 & 0.66839 & 13.37 & 88.90 \\
5 & 0.55519 & 11.10 & 100.00
\end{tabular}

Векторам на биплоте соответствуют проекции единичных векторов, направленных вдоль осей систем координат пар исходных данных, на плоскость выделенных пар главных компонент. Сами вектора часто называются нагрузками [5]. Точками показаны компоненты так называемой матрицы счетов, т. е. проекции исходных данных на плоскости пар соответствующих главных компонент. На рис. 3-5 приведены счета и нагрузки для пары главных компонент РC1 и РC2, PC3 и PC1, PC3 и РC2 соответственно.

Из рис. 3, 4 следует, что все вектора действуют однонаправленно и имеют положительную проекцию на ось первого фактора совместно с вектором сахарного диабета. Из рис. 5 следует, что комбинация главных компонент 2 и 3 не позволяет выявить положительной связи между заболеваемостью сахарным диабетом и факторами урбанизации. Однако вклад в суммарную дисперсию фактора 3 мал: как следует из табл. 3, он не превышает $15 \%$, поэтому анализ этого рисунка не является решающим. Поэтому все сказанное выше свидетельствует о положительной связи факторов, которым соответствуют вектора, на заболеваемость DM1. Эти вектора, однако, действуют только на жителей ряда регионов РФ, положение которых отмечено на правой полуплоскости точками. Регионы левой полуплоскости, по-видимому, подвержены действию других факторов, таких как, например, генетическая предрасположенность, которые не выявлялись и не анализировались в настоящей работе.

Поскольку среди факторов присутствуют дороги с твердым покрытием и число автобусов, их влияние на возникновение и развитие DM1 можно свести к действию дорожной пыли. В частности, углеродная составляющая пыли может образовываться из-за горения шин на участках торможения-разгона транспорта при движении по трассе, в основном при резком торможении и наборе скорости. Помимо прямого воздействия пыли на организм вследствие ее абсорбции организмом и последующем метаболизме, она может оказывать отрицательное воздействие на иммунные реакции организма. Кроме того, влияние углеродной составляющей может проявляться опосредованно, через поглощение света Солнца в ультрафиолетовом спектральном диапазоне. 
Например, полициклические ароматические углеводороды входят в качестве компонентов дорожной пыли $[7,9,10]$. Попадая в атмосферу и находясь там во взвешенном состоянии, они способны поглощать УФ излучение с длинами волн, которые вызывают фотохимические реакции синтеза витамина D кожей человека [3,9]. Уменьшение инсоляции, в свою очередь, может способствовать проявлению D-витаминной недостаточности и по современным данным проявлению симптомокомплекса аутоиммунных патологий, в том числе DM1 [9]. Нельзя также исключить аналогичного действия на солнечное излучение других факторов урбанизации, исследованных в настоящей работе. Однако поскольку в литературе ранее подробный анализ систематически не проводился, более подробное рассмотрение этого вопроса следовало бы провести в ближайшем будущем.

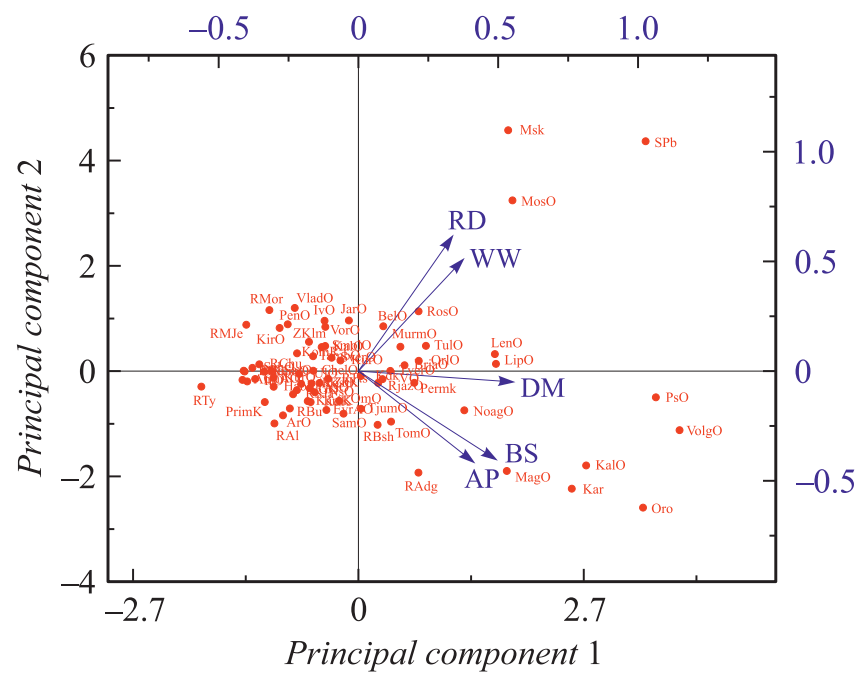

Рис. 3. Рисунок счетов и нагрузок для пары главных компонент РC1 и РC2.

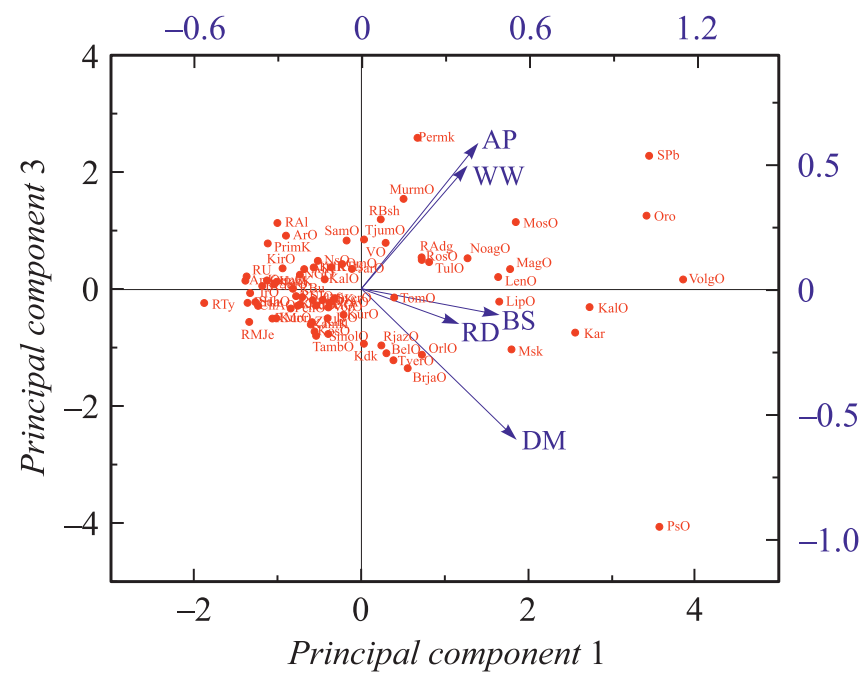

Рис. 4. Рисунок счетов и нагрузок для пары главных компонент РС3 и РС1.

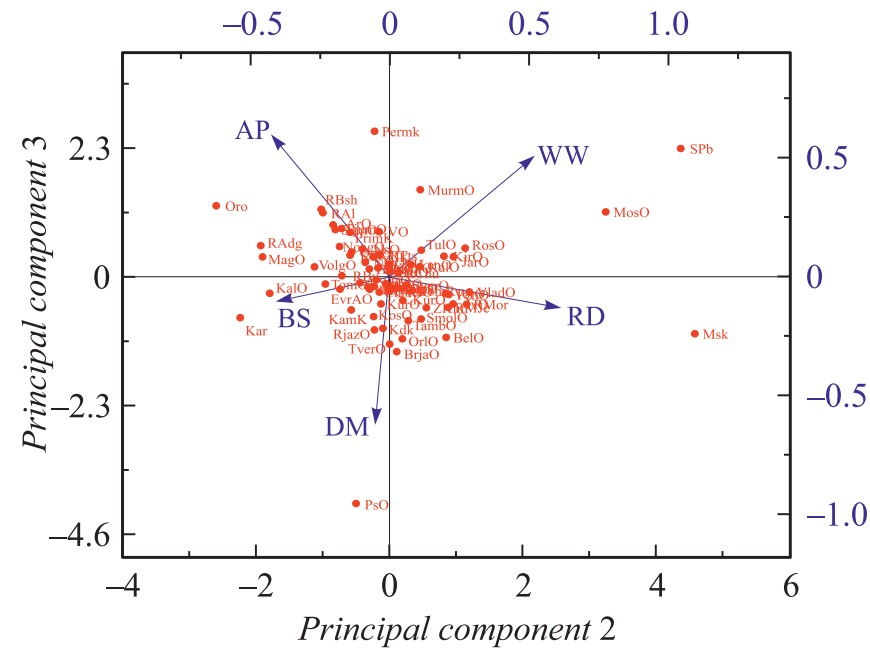

Рис. 5. Рисунок счетов и нагрузок для пары главных компонент РС3 и РС2.

\section{Выводы}

Проанализирована связь между факторами урбанизации с DM1. Выполненный анализ позволил построить гипотетическую физическую модель, позволяющую связать эти факторы с уменьшением инсоляции пациентов ультрафиолетовым излучением. Также данная модель может способствовать широкому внедрению в практику для разработки клинико-диагностических критериев и принятия организационно-управленческих решений для профилактики заболеваемости DM1.

\section{Финансирование работы}

Работа выполнена на кафедре Организации Здравоохранения и Медицинского права СПбГУ и ФТИ им. А.Ф. Иоффе в рамках бюджетного финансирования.

Работа поддержана грантом Правительства РФ (договор 14.W03.31.0009 от 13.02.2017 г.) о выделении гранта для государственной поддержки научных исследований, проводимых под руководством ведущих ученых. Авторы внесли равный вклад в написание работы.

\section{Конфликт интересов}

Авторы заявляют, что у них нет конфликта интересов.

\section{Список литературы}

[1] Berridge M.J. // Biochem. J. 2017. Vol. 474. N 8. P. 1321-1332. DOI: 10.1042/ BCJ20170042

[2] Terenetskaya I.P. // Integr. Mol. Med. 2018. Vol. 5. N 2. P. 1-5. DOI: 10.15761/IMM.1000327 
[3] Szalecki M., Wysocka-Mincewicz M., Ramotowska A., Mazur A., Lisowicz L., Beń-Skowronek I., Sieniawska J., Klonowska B., Charemska D., Nawrotek J., Jatowiec I., Bossowski A., Jamiotkowska M., Pyrżak B., Miszkurka G., Szypowska A. // Res. Rev. 2018. Vol. 34. N 2.

DOI: $10.1002 / \mathrm{dmrr} .2962$

[4] Федеральная служба государственной статистики (Росстат) статистический сборник регионы России. Социально-экономические показатели. 2009, 2012, 2014, 2016. М.: Росстат, 990, 1090, 1180, 1200 с.

[5] Principal Component Analysis / I.T. Jolliffe. Second Edition, NY: Springer, 2002. 487 p. DOI: $10.1007 /$ b98835

[6] Verstraete L., Leger A. // Astron. Astrophys. 1996. Vol. 240. N 1. P. 55-73. DOI: $10.1007 / \mathrm{BF} 00640196$

[7] Леванчук А.В. // Гигиена и санитария. 2014. Т. 93 Вып.6. C. 17-21. [Levanchuk A.V. // Hygiene and Sanitation. 2014. Vol. 93. N 6. P. 17-21.]

[8] Pollard K.M., Cavi D.M., Toomey C.B., Hultman P., Kono D.H. // BBA-General Subjects. 2019. Vol. 1863. N 12. P. 129299. DOI:10.1016/j.cej.2018.08.019

[9] Руководство по аутоиммунным заболеваниям для врачей общей практики / Под ред. И. Шенфельда, П.Л. Мерони, Л.П. Чурилова. СПб: Медкнига „ЭЛБИ“, 2017. 416 c. [Guide in Autoimmune Diseases for General medical Practice. Y. Shoenfeld, P.L. Meroni, L.P. Churilov. Saint Petersburg: ELBI-Medkniga, 2017. 416 c.]

[10] Копытенкова О.И., Шилова ЕА., Сазонова А.М. // Наука. 2015. T. 7. Вып. 1. С. 26-43. DOI: $10.15862 / 103 T V N 115$ [Kopytenkova O.I., Shilova E.A., Sazonova A.M. // Science. 2015. Vol. 7. N 1. P. 26-43.] 